\title{
Metabolome analysis of esophageal cancer tissues using capillary electrophoresis-time-of-flight mass spectrometry
}

\author{
MASANORI TOKUNAGA ${ }^{1,2}$, KENJIRO KAMI $^{3}$, SOJI OZAWA $^{2}$, JUNYA OGUMA $^{2}$, AKIHITO KAZUNO $^{2}$, \\ HAYATO MIYACHI $^{4}$, YOSHIAKI OHASHI ${ }^{3}$, MASATOSHI KUSUHARA ${ }^{5}$ and MASANORI TERASHIMA ${ }^{1}$ \\ ${ }^{1}$ Division of Gastric Surgery, Shizuoka Cancer Center, Shizuoka 411-8777; ${ }^{2}$ Department of Gastroenterological Surgery, \\ Tokai University School Medicine, Isehara, Kanagawa 259-1193; ${ }^{3}$ Human Metabolome Technologies, Inc., \\ Tsuruoka, Yamagata 997-0052; ${ }^{4}$ Department of Laboratory Medicine, Tokai University School Medicine, \\ Isehara, Kanagawa 259-1193; ${ }^{5}$ Regional Resources Division, Shizuoka Cancer Center, Shizuoka 411-8777, Japan
}

Received December 12, 2017; Accepted March 15, 2018

DOI: $10.3892 /$ ijo.2018.4340

\begin{abstract}
Reports of the metabolomic characteristics of esophageal cancer are limited. In the present study, we thus conducted metabolome analysis of paired tumor tissues (Ts) and non-tumor esophageal tissues (NTs) using capillary electrophoresis time-of-flight mass spectrometry (CE-TOFMS). The Ts and surrounding NTs were surgically excised pairwise from 35 patients with esophageal cancer. Following tissue homogenization and metabolite extraction, a total of 110 compounds were absolutely quantified by CE-TOFMS. We compared the concentrations of the metabolites between Ts and NTs, between pT1 or pT2 (pT1-2) and pT3 or pT4 (pT3-4) stage, and between node-negative $\left(\mathrm{pN}^{-}\right)$and node-positive $\left(\mathrm{pN}^{+}\right)$samples. Principal component analysis and hierarchical clustering analysis revealed clear metabolomic differences between Ts and NTs. Lactate and citrate levels in Ts were significantly higher $(\mathrm{P}=0.001)$ and lower $(\mathrm{P}<0.001)$, respectively, than those in NTs, which corroborated with the Warburg effect in Ts. The concentrations of most amino acids apart from glutamine were higher in Ts than in NTs, presumably due to hyperactive glutaminolysis in Ts. The concentrations of malic acid $(\mathrm{P}=0.015)$ and citric acid $(\mathrm{P}=0.008)$ were significantly lower in pT3-4 than in pT1-2, suggesting the downregulation of tricarboxylic acid (TCA) cycle activity in pT3-4. On the whole, in this study, we demonstrate significantly different metabolomic characteristics between tumor and non-tumor tissues and identified a novel set of metabolites that were strongly associated with the degree of tumor progression. A further understanding of cancer metabolomics may enable
\end{abstract}

Correspondence to: Professor Soji Ozawa, Department of Gastroenterological Surgery, Tokai University School Medicine, 143 Shimokasuya, Isehara, Kanagawa 259-1193, Japan

E-mail: sozawa@tokai.ac.jp

Key words: esophageal cancer, mass spectrometry, metabolome, Warburg effect, tumor progression the selection of more appropriate treatment strategies, thereby contributing to individualized medicine.

\section{Introduction}

Esophageal cancer is the eighth most common type of cancer and the sixth leading cause of cancer-related mortality worldwide. It is frequently observed in East Asia (1). The clinicopathological characteristics of esophageal cancer have been investigated and clarified. Pathological tumor depth, nodal status and stage are known to be strongly associated with the survival outcome, which has been recently improved with advancements in multimodal treatments (2). However, the long-term survival outcome remains dismal, and the 5-year survival rate of patients with potentially curable advanced esophageal cancer has been reported to be only $34-55 \%$, according to recent randomized controlled trials $(3,4)$. To improve this poor survival outcome, appropriate treatment strategies tailored for each individual patient are warranted. To achieve this, the biological characteristics and causal factors of the survival outcome require clarification. Recently, it has been reported that the progression of the disease may affect the biological activity of some metabolites $(5,6)$.

Metabolome analysis may enable us to understand tumorspecific metabolic characteristics, which would facilitate the discovery of novel anticancer drug targets and therapeutic strategies (7). Thus far, comparative metabolomic profiling has been conducted for several cancer types, such as gastric, lung, prostate, or colorectal cancers $(7,8)$. Metabolomic profiles of esophageal cancer have also been investigated using blood samples (5,9-12) or paired tumor and non-tumor tissues $(5,13,14)$. Metabolomic analysis using blood is preferable for the identification of tumor markers by comprehensive analysis; however, it does not reflect the microenvironment of the tumor, which can only be clarified using tissue samples. In addition, the majority of previous studies have used either nuclear magnetic resonance (NMR) $(13,14)$ or gas chromatography-mass spectrometry (GC-MS) (15) for analysis. However, capillary electrophoresis-mass spectrometry (CE-MS), which is specialized for the analysis of ionic metabolites and thus may lead to the identification of novel metabolic properties of 
cancer, has rarely been used for the metabolomic analysis of paired tumor and non-tumor tissues. Furthermore, the associations between metabolomic characteristics and advancement of the disease or survival outcome have rarely been investigated and remain unclear. Although Wang et al clarified the associations between metabolomic characteristics and tumor stages, only 45 metabolites were identified by NMR analysis, and the associations between metabolomic characteristics and other clinical factors were not investigated (14).

Therefore, the aim of the present study was to clarify the potential association between pathological disease status and metabolome profiles of tissues in patients with esophageal cancer. We also investigated the differences in metabolomic characteristics between tumor and non-tumor tissues from patients with esophageal cancer.

\section{Patients and methods}

Patient characteristics. The present study was designed as a single-center, prospective observational study. The institutional review board of Tokai University (Isehara, Japan) approved the study protocol, which had the following inclusion criteria: i) Patients with histologically confirmed adenocarcinoma or squamous cell carcinoma of the esophagus undergoing curative esophagectomy; ii) the size of the primary tumor large enough to obtain $1 \mathrm{~g}$ of tumor tissue without affecting the pathological examination; iii) an age of 20 years or older; and iv) written informed consent. Pathological tumor depth, nodal status and stage were assigned according to the Japanese Classification of Esophageal Cancer, 11th edition (16).

Between May, 2012 and October, 2013, a total of 35 patients were enrolled in the present study, and 35 pairs of tumor (Ts) and non-tumor (NTs) esophageal tissues were obtained. The characteristics and pathological findings of the patients are presented in Table I. Neoadjuvant chemotherapy was administered to 17 patients, and the majority of patients underwent subtotal esophagectomy. The surgery was curative (R0) in 24 patients, and resulted in microscopic residual disease (R1) in 7 patients and macroscopic residual disease (R2) in 4 patients. The disease was advanced in the majority of the patients, and the pathological stage was III or IVa in $77 \%$ of the patients.

Tissue sampling and metabolite extraction. Tumor and surrounding tissues were surgically resected from each of the 35 patients with esophageal cancer immediately following esophagectomy. The resected tissue samples were promptly frozen in liquid nitrogen and stored at $-80^{\circ} \mathrm{C}$ until metabolite extraction. To inactivate enzymes, $\sim 50 \mathrm{mg}$ of frozen tissue was immersed into $1,500 \mu \mathrm{l}$ of $50 \%$ acetonitrile/Milli-Q water containing internal standards [H3304-1002; Human Metabolome Technologies (HMT), Tsuruoka, Japan] at $0^{\circ} \mathrm{C}$. The tissue was homogenized 3 times at 1,500 rpm for $120 \mathrm{sec}$ using a tissue homogenizer (Microsmash MS100R; Tomy Digital Biology Co., Ltd., Tokyo, Japan) before the homogenate was centrifuged at $2,300 \mathrm{x} \mathrm{g}$ and $4^{\circ} \mathrm{C}$ for $5 \mathrm{~min}$. Subsequently, $800 \mu \mathrm{l}$ of the the upper aqueous layer were centrifugally filtered through a Millipore 5,000-Da cut-off filter at 9,100 $\mathrm{xg}$ and $4^{\circ} \mathrm{C}$ for $120 \mathrm{~min}$ to remove proteins. The filtrate was centrifugally concentrated and re-suspended in $50 \mu \mathrm{l}$ of Milli-Q water for
Table I. Characteristics of patients with adenocarcinoma or squamous cell carcinoma (SCC) of the esophagus.

Sex, $\mathrm{n}$
Male
Female
Age, years
Median
Range
Performance status, $\mathrm{n}$
0
1
Neoajuvant chemotherapy, $\mathrm{n}$
+

Histology

Well differientated SCC $\quad 13$

Moderately differientated SCC 18

Poorly differientated SCC 4

Tumor diameter (mm)

Median

Range

Lymphatic invasion

$+$

Vascular invasion

-

$+$

Tumor depth

T1

$\mathrm{T} 2$

T3

T4

Nodal status

N0

8

N1

N2

N3

N4

Number of lymph node metastases

Median

Range

Stage

I

II

0

8

III

22

IVa

Curability

R0

$\mathrm{R} 1$

$\mathrm{R} 2$ 
capillary electrophoresis time-of-flight mass spectrometry (CE-TOFMS) analysis.

Metabolome analysis. Metabolome analysis was conducted by the Basic Scan package from HMT using CE-TOFMS based on previously described methods $(17,18)$. Briefly, CE-TOFMS analysis was conducted using an Agilent CE capillary electrophoresis system equipped with an Agilent 6210 time-of-flight mass spectrometer (Agilent Technologies, Waldbronn, Germany). The systems were controlled by Agilent G2201AA ChemStation software version B.03.01 for CE (Agilent Technologies). The spectrometer was scanned from 50 to $1,000 \mathrm{~m} / \mathrm{z}$, and peaks were extracted using MasterHands automatic integration software (Keio University, Tsuruoka, Yamagata, Japan) to obtain peak information including $m / z$, peak area, and migration time (MT) (19). Signal peaks corresponding to isotopomers, adduct ions and other product ions of known metabolites were excluded, and based on their $m / z$ values with the MTs, remaining peaks were annotated according to the HMT's proprietary metabolite database. The areas of the annotated peaks were normalized based on internal standard levels and sample quantities to obtain relative levels of each metabolite.

Statistical analysis. Hierarchical cluster analysis (HCA) and principal component analysis (PCA) were performed using the proprietary software from HMT, PeakStat and SampleStat, respectively. Detected metabolites were plotted on metabolic pathway maps using VANTED software (20). All continuous data, including age, tumor diameter and the number of lymph node metastases, are presented as medians (range) and were analyzed by the Wilcoxon rank-sum test. A value of $\mathrm{P}<0.05$ was considered to indicate a statistically significant difference. For any compound that was not detected in a tissue from the subjects, half of the minimum value of the measured compound replaced the missing data. Metabolomic profiles were compared between i) tumor and non-tumor tissues to elucidate differences in metabolomic profiles between them; ii) patients with $\mathrm{T} 1$ or $\mathrm{T} 2$ disease (pT1-2) and those with $\mathrm{T} 3$ or T4 disease (pT3-4); and iii) patients with node-negative $\left(\mathrm{pN}^{-}\right)$ and node-positive $\left(\mathrm{pN}^{+}\right)$disease.

\section{Results}

Metabolomic characteristics between Ts and NTs. The metabolome data were normalized based on their z-values and used for PCA and HCA. The PCA plot presented in Fig. 1 shows a clear separation between NTs and Ts along the PC1 axis, indicating an apparently different metabolomic profile between NTs and Ts. The PCA plot also indicates a higher heterogeneity in the metabolomic profiles of Ts than of NTs. According to the HCA presented in Fig. 2, approximately two thirds of all the measured metabolites were higher in Ts than in NTs.

Metabolites measured in the present analysis were visualized on a metabolome-wide pathway map (available upon requested), and Fig. 3 illustrates the pathway map of the tricarboxylic acid (TCA) cycle. A total of 110 compounds were measured, and 99 compounds were absolutely quantified in this study (Table II). Of these, the concentrations of as many as 58 compounds were statistically significantly different between

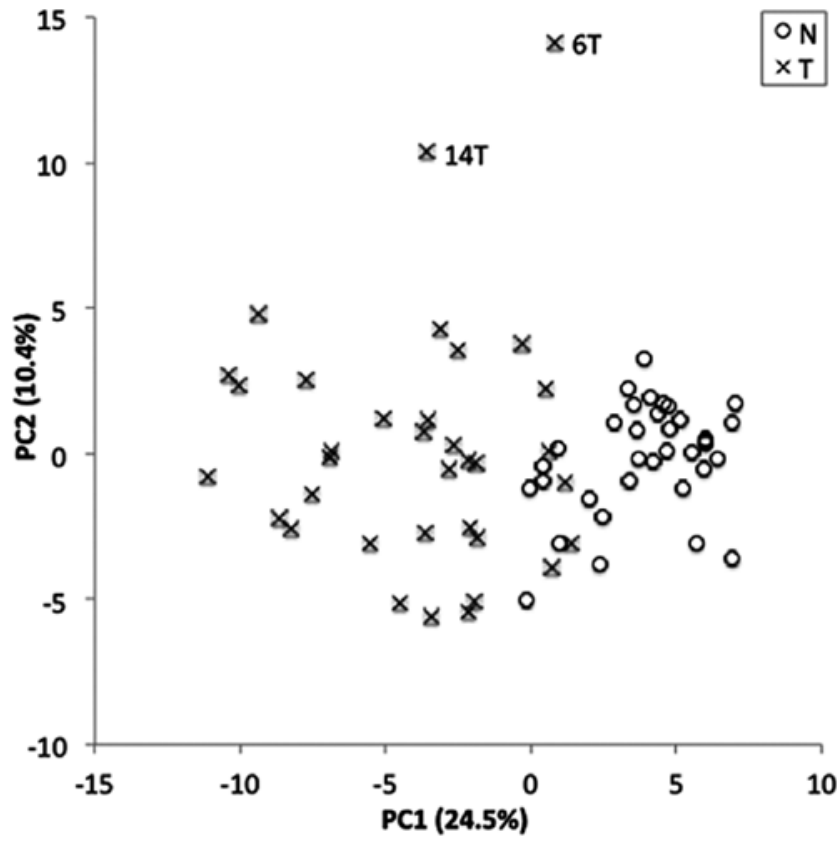

Figure 1. Principal component analysis plot.

Ts and NTs $(\mathrm{P}<0.05)$. Fig. 4 and Table II illustrate all the measured metabolites in this study listed in descending order and based on Ts/NTs ratios. The concentrations of most amino acids apart from glutamine were significantly higher in Ts than in NTs (Fig. 5). In addition, as shown in Table II, the levels of nucleoside triphosphates [adenosine triphosphate (ATP), cytidine triphosphate (CTP), guanosine-5'-triphosphate (GTP) and uridine-5'-triphosphate (UTP)] were statistically significantly lower in Ts, whereas those of nucleoside monophosphates, such as guanosine monophosphate (GMP) were much higher. The concentrations of isocitric acid, $c i s$-aconitic acid and citric acid, which are the upstream TCA cycle intermediates, were significantly lower in Ts than in NTs, while the lactic acid level was significantly higher in Ts.

Metabolomics with pathological tumor depth $(p T)$ and pathological nodal status $(p N)$ relevance. Tumor depth is known to be associated with the expression levels of glucose transporter (21) and several glycolytic enzymes, such as hexokinase 2 (22) and pyruvate kinase M2 (23). Thus, in this study, the tumor concentrations of the quantified metabolites were compared between pT1-2 and pT3-4 tumor tissues. Table III presents a list of metabolites of which the concentrations were at least 1.5-fold higher (7 metabolites) or lower (21 metabolites) in pT3-4 than in pT1-2). The concentrations of glycolytic and pentose phosphate pathway intermediates were higher overall in subjects with advanced disease (pT3-4), and the ratios of glucose 1-phosphate, ribose 5-phosphate and ribulose 5-phosphate were $1.92,1.58$ and 1.56 , respectively, and $>1.5$-fold higher in pT3-4 than pT1-2. By contrast, the concentrations of malic acid and citric acid, also TCA cycle intermediates, and most nucleotides were significantly lower in pT3-4 than in pT1-2, possibly rationalizing relatively hypoxic microenvironment of advanced tumor tissues (24). Moreover, adenine-, cytidine- and uridine-nucleotide concentrations were lower in pT3-4 than in pT1-2 tumors, while the glutathione and 


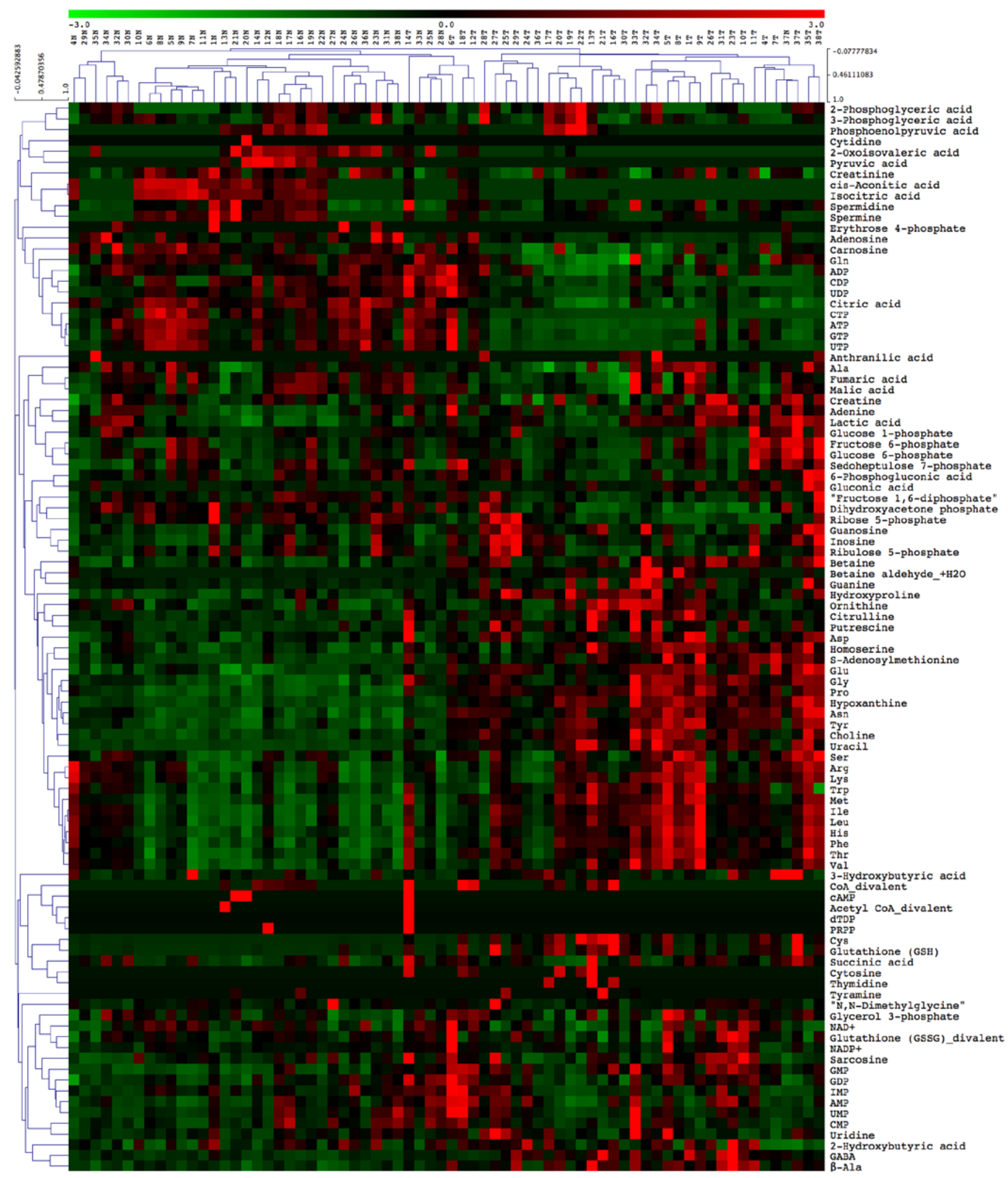

Figure 2. Heatmap of hierarchical cluster analysis.

cysteine levels were higher in pT3-4 than in pT1-2, with ratios being 1.80 and 3.36, respectively (Table III).

Metastatic alterations seemingly affect the balance of energy metabolism between glycolysis and oxidative phosphorylation $(25,26)$. Jin et al identified a series of serum metabolites, such as valine and GABA that differ significantly in patients with esophageal squamous cell carcinoma with or without lymph node metastasis using a metabolomics approach (27). In this study, we thus investigated whether there was any metabolic difference in primary tumor tissues with or without metastasis. Table IV lists the metabolites the concentrations of which were at least 1.5-fold higher 


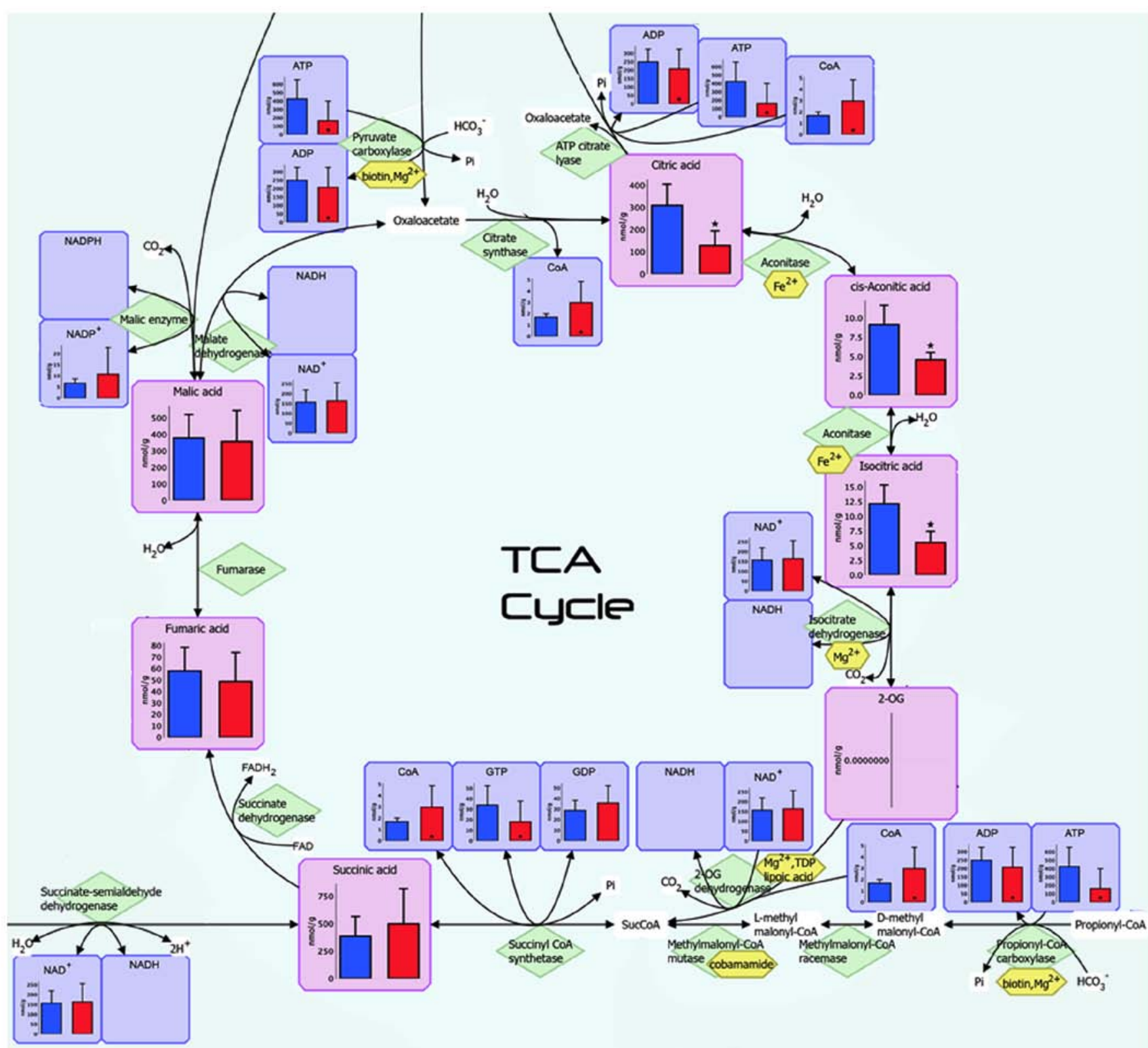

Figure 3. Metabolic pathway map comparing metabolite concentrations of tumor and non-tumor tissues. Blue bars indicate the concentration of non-tumor tissue, and red bars indicate that of tumor tissue.

( 2 metabolites) or lower (18 metabolites) in $\mathrm{pN}^{+}$than in $\mathrm{pN}^{-}$. $\mathrm{N}, \mathrm{N}$-dimethylglycine, isocitric acid, fructose 1,6-diphosphate and aspartic acid were statistically significantly lower in the $\mathrm{pN}^{+}$than the $\mathrm{pN}^{-}$tumor tissues. Of note, many nucleotide concentrations including ATP, GTP, CTP and UTP tended to be lower in the $\mathrm{pN}^{+}$than $\mathrm{pN}^{-}$tumors, although the difference was not statistically significant, with the exception of IMP and UMP.

\section{Discussion}

Thus far, metabolomic differences between tumor and nontumor tissues have been investigated elsewhere in various types of cancer $(7,8,13,14)$. The results of the present study not only demonstrated the basal metabolomic differences between esophageal tumor and non-tumor tissues, but also identified intriguing associations of metabolites with the degree of tumor advancement and with the presence or absence of lymph node metastasis.

Statistical significances between Ts and NTs were found in 58 out of 110 compounds, including isocitric acid, cis-aconitic acid, and citric acid, which were significantly lower in Ts than NTs, and lactic acid, which was significantly higher in Ts. These features suggest the upregulation of glycolysis and lactate formation, and the downregulation of the flux into the TCA cycle, and thus corroborate the hallmark of cancer metabolism i.e., the Warburg effect $(7,28)$.

In the present study, the tumor concentrations of all amino acids apart from glutamine were higher than their non-tumor counterparts. Amino acid synthesis may be globally enhanced; however, this does not explain the significantly higher concentrations of even essential amino acids. The data thus possibly imply the hyperactivity of amino acid transporters (29-31) or autophagic protein degradation (32), both of which contribute 
Table II. Concentrations of compounds (listed in descending order based on Ts/NTs ratios) in Ts and NTs.

\begin{tabular}{|c|c|c|c|c|c|c|c|}
\hline Name of compound & NTs & Ts & Ratio (Ts/NTs) & P-value & ND/all (70) & ND/NTs (35) & ND/Ts (35) \\
\hline 2-Oxoglutaric acid & 0.000 & 0.000 & NA & NA & 70 & 35 & 35 \\
\hline cGMP & 0.000 & 0.000 & NA & NA & 70 & 35 & 35 \\
\hline dATP & 0.000 & 0.000 & NA & NA & 70 & 35 & 35 \\
\hline $\mathrm{dCTP}$ & 0.000 & 0.000 & NA & NA & 70 & 35 & 35 \\
\hline dTMP & 0.000 & 0.000 & NA & NA & 70 & 35 & 35 \\
\hline dTTP & 0.000 & 0.000 & NA & NA & 70 & 35 & 35 \\
\hline Glyceraldehyde 3-phosphate & 0.000 & 0.000 & NA & NA & 70 & 35 & 35 \\
\hline Glycolic acid & 0.000 & 0.000 & NA & NA & 70 & 35 & 35 \\
\hline Glyoxylic acid & 0.000 & 0.000 & NA & NA & 70 & 35 & 35 \\
\hline Malonyl CoA_divalent & 0.000 & 0.000 & NA & NA & 70 & 35 & 35 \\
\hline Thymine & 0.000 & 0.000 & NA & NA & 70 & 35 & 35 \\
\hline Cys & 2.737 & 182.622 & 66.72 & $<0.001$ & 19 & 16 & 3 \\
\hline Glutathione (GSH) & 55.380 & 839.397 & 15.16 & $<0.001$ & 13 & 10 & 3 \\
\hline Uracil & 21.761 & 168.667 & 7.75 & $<0.001$ & 1 & 1 & 0 \\
\hline Betaine aldehyde_$+\mathrm{H}_{2} \mathrm{O}$ & 0.094 & 0.674 & 7.20 & $<0.001$ & 45 & 31 & 14 \\
\hline Hypoxanthine & 170.444 & 746.527 & 4.38 & $<0.001$ & 0 & 0 & 0 \\
\hline S-Adenosylmethionine & 12.254 & 40.406 & 3.30 & $<0.001$ & 0 & 0 & 0 \\
\hline Putrescine & 36.506 & 119.342 & 3.27 & $<0.001$ & 0 & 0 & 0 \\
\hline Gluconic acid & 28.168 & 87.567 & 3.11 & $<0.001$ & 0 & 0 & 0 \\
\hline Guanine & 15.205 & 43.218 & 2.84 & 0.226 & 2 & 0 & 2 \\
\hline GMP & 29.556 & 76.026 & 2.57 & $<0.001$ & 0 & 0 & 0 \\
\hline Met & 97.882 & 243.854 & 2.49 & $<0.001$ & 0 & 0 & 0 \\
\hline GABA & 12.250 & 30.295 & 2.47 & $<0.001$ & 0 & 0 & 0 \\
\hline Tyramine & 0.166 & 0.408 & 2.46 & 0.404 & 64 & 33 & 31 \\
\hline Choline & 161.699 & 395.925 & 2.45 & $<0.001$ & 0 & 0 & 0 \\
\hline Homoserine & 0.843 & 2.001 & 2.37 & $<0.001$ & 12 & 10 & 2 \\
\hline Pro & 377.514 & 890.493 & 2.36 & $<0.001$ & 0 & 0 & 0 \\
\hline Citrulline & 30.403 & 69.160 & 2.27 & $<0.001$ & 0 & 0 & 0 \\
\hline Asn & 124.410 & 275.376 & 2.21 & $<0.001$ & 0 & 0 & 0 \\
\hline Tyr & 140.516 & 307.546 & 2.19 & $<0.001$ & 0 & 0 & 0 \\
\hline Hydroxyproline & 28.391 & 58.942 & 2.08 & $<0.001$ & 0 & 0 & 0 \\
\hline$\beta$-Ala & 36.973 & 75.260 & 2.04 & $<0.001$ & 0 & 0 & 0 \\
\hline Betaine & 47.056 & 94.012 & 2.00 & $<0.001$ & 0 & 0 & 0 \\
\hline Ile & 265.350 & 510.584 & 1.92 & $<0.001$ & 0 & 0 & 0 \\
\hline Leu & 492.049 & 914.861 & 1.86 & $<0.001$ & 0 & 0 & 0 \\
\hline Phe & 229.967 & 418.200 & 1.82 & $<0.001$ & 0 & 0 & 0 \\
\hline Asp & 403.697 & 729.062 & 1.81 & $<0.001$ & 0 & 0 & 0 \\
\hline Guanosine & 16.504 & 28.872 & 1.75 & 0.001 & 0 & 0 & 0 \\
\hline Gly & 1615.687 & 2817.176 & 1.74 & $<0.001$ & 0 & 0 & 0 \\
\hline Glu & 1862.762 & 3242.047 & 1.74 & $<0.001$ & 0 & 0 & 0 \\
\hline CoA_divalent & 0.575 & 0.984 & 1.71 & 0.463 & 54 & 28 & 26 \\
\hline His & 222.272 & 375.296 & 1.69 & $<0.001$ & 0 & 0 & 0 \\
\hline Val & 564.942 & 937.011 & 1.66 & $<0.001$ & 0 & 0 & 0 \\
\hline $\mathrm{NADP}^{+}$ & 6.226 & 10.256 & 1.65 & 0.142 & 5 & 3 & 2 \\
\hline Inosine & 118.058 & 193.143 & 1.64 & $<0.001$ & 0 & 0 & 0 \\
\hline Adenine & 0.945 & 1.510 & 1.60 & $<0.001$ & 0 & 0 & 0 \\
\hline Thr & 540.238 & 841.303 & 1.56 & $<0.001$ & 0 & 0 & 0 \\
\hline Trp & 56.496 & 87.291 & 1.55 & $<0.001$ & 0 & 0 & 0 \\
\hline Cytosine & 0.075 & 0.113 & 1.50 & 0.011 & 64 & 35 & 29 \\
\hline Ornithine & 102.318 & 150.059 & 1.47 & 0.010 & 0 & 0 & 0 \\
\hline Uridine & 50.310 & 72.303 & 1.44 & 0.108 & 0 & 0 & 0 \\
\hline AMP & 234.908 & 327.723 & 1.40 & 0.170 & 0 & 0 & 0 \\
\hline
\end{tabular}


Table II. Continued.

\begin{tabular}{|c|c|c|c|c|c|c|c|}
\hline Name of compound & NTs & Ts & Ratio (Ts/NTs) & P-value & ND/all (70) & ND/NTs (35) & ND/Ts (35) \\
\hline Sarcosine & 11.929 & 16.584 & 1.39 & 0.091 & 0 & 0 & 0 \\
\hline Fructose 6-phosphate & 13.418 & 18.209 & 1.36 & 0.196 & 7 & 5 & 2 \\
\hline Lactic acid & 30047.277 & 40727.323 & 1.36 & 0.001 & 0 & 0 & 0 \\
\hline UMP & 39.626 & 53.320 & 1.35 & 0.320 & 0 & 0 & 0 \\
\hline Lys & 728.484 & 958.409 & 1.32 & 0.002 & 0 & 0 & 0 \\
\hline Succinic acid & 385.179 & 497.439 & 1.29 & 0.140 & 0 & 0 & 0 \\
\hline Arg & 396.418 & 507.514 & 1.28 & 0.010 & 0 & 0 & 0 \\
\hline GDP & 28.004 & 35.686 & 1.27 & 0.036 & 1 & 1 & 0 \\
\hline Glycerol 3-phosphate & 211.332 & 268.640 & 1.27 & 0.054 & 0 & 0 & 0 \\
\hline Sedoheptulose 7-phosphate & 19.238 & 24.436 & 1.27 & 0.095 & 0 & 0 & 0 \\
\hline Ser & 467.826 & 590.577 & 1.26 & 0.077 & 0 & 0 & 0 \\
\hline 3-Hydroxybutyric acid & 287.678 & 355.980 & 1.24 & 0.051 & 0 & 0 & 0 \\
\hline Glucose 1-phosphate & 25.489 & 31.150 & 1.22 & 0.362 & 0 & 0 & 0 \\
\hline IMP & 31.863 & 37.742 & 1.18 & 0.506 & 1 & 0 & 1 \\
\hline Glutathione (GSSG)_divalent & 560.285 & 663.095 & 1.18 & 0.674 & 0 & 0 & 0 \\
\hline Glucose 6-phosphate & 84.783 & 99.749 & 1.18 & 0.870 & 0 & 0 & 0 \\
\hline Thymidine & 1.100 & 1.273 & 1.16 & 0.082 & 67 & 35 & 32 \\
\hline CMP & 10.600 & 12.012 & 1.13 & 0.664 & 7 & 5 & 2 \\
\hline 2-Hydroxybutyric acid & 115.702 & 130.960 & 1.13 & 0.344 & 0 & 0 & 0 \\
\hline Ribulose 5-phosphate & 33.894 & 37.243 & 1.10 & 0.753 & 0 & 0 & 0 \\
\hline Spermidine & 17.565 & 18.966 & 1.08 & 0.326 & 0 & 0 & 0 \\
\hline Creatine & 1608.615 & 1719.858 & 1.07 & 0.907 & 0 & 0 & 0 \\
\hline Anthranilic acid & 0.233 & 0.249 & 1.07 & 0.241 & 63 & 33 & 30 \\
\hline 6-Phosphogluconic acid & 15.030 & 15.899 & 1.06 & 0.318 & 3 & 1 & 2 \\
\hline 2-Phosphoglyceric acid & 8.473 & 8.918 & 1.05 & 0.812 & 21 & 10 & 11 \\
\hline$N, N$-Dimethylglycine & 3.640 & 3.802 & 1.04 & 0.398 & 3 & 1 & 2 \\
\hline PRPP & 1.423 & 1.486 & 1.04 & 1.000 & 68 & 34 & 34 \\
\hline $\mathrm{NAD}^{+}$ & 156.517 & 163.076 & 1.04 & 0.815 & 0 & 0 & 0 \\
\hline dTDP & 0.656 & 0.675 & 1.03 & 0.331 & 69 & 35 & 34 \\
\hline Phosphoenolpyruvic acid & 4.217 & 4.285 & 1.02 & 0.947 & 50 & 25 & 25 \\
\hline Ala & 1740.699 & 1756.570 & 1.01 & 0.788 & 0 & 0 & 0 \\
\hline Acetyl CoA_divalent & 0.411 & 0.414 & 1.01 & 1.000 & 68 & 34 & 34 \\
\hline cAMP & 0.425 & 0.422 & 0.99 & 0.592 & 67 & 33 & 34 \\
\hline Fructose 1,6-diphosphate & 66.853 & 66.265 & 0.99 & 0.072 & 1 & 1 & 0 \\
\hline Cytidine & 3.452 & 3.356 & 0.97 & 0.331 & 69 & 34 & 35 \\
\hline Malic acid & 377.443 & 357.165 & 0.95 & 0.362 & 0 & 0 & 0 \\
\hline 3-Phosphoglyceric acid & 74.824 & 69.761 & 0.93 & 0.247 & 0 & 0 & 0 \\
\hline Creatinine & 57.646 & 51.442 & 0.89 & 0.051 & 0 & 0 & 0 \\
\hline ADP & 248.784 & 207.315 & 0.83 & 0.011 & 0 & 0 & 0 \\
\hline Fumaric acid & 57.785 & 47.315 & 0.82 & 0.019 & 1 & 0 & 1 \\
\hline Gln & 2277.779 & 1703.272 & 0.75 & $<0.001$ & 0 & 0 & 0 \\
\hline Ribose 5-phosphate & 11.736 & 7.814 & 0.67 & $<0.001$ & 11 & 1 & 10 \\
\hline UDP & 42.200 & 27.604 & 0.65 & $<0.001$ & 0 & 0 & 0 \\
\hline Carnosine & 2.419 & 1.397 & 0.58 & $<0.001$ & 4 & 0 & 4 \\
\hline Dihydroxyacetone phosphate & 28.798 & 16.384 & 0.57 & $<0.001$ & 5 & 0 & 5 \\
\hline Pyruvic acid & 24.610 & 13.810 & 0.56 & 0.011 & 61 & 27 & 34 \\
\hline GTP & 34.037 & 17.962 & 0.53 & $<0.001$ & 0 & 0 & 0 \\
\hline CDP & 7.051 & 3.378 & 0.48 & $<0.001$ & 14 & 6 & 8 \\
\hline Spermine & 10.261 & 4.708 & 0.46 & 0.151 & 19 & 10 & 9 \\
\hline Citric acid & 308.711 & 128.575 & 0.42 & $<0.001$ & 0 & 0 & 0 \\
\hline Adenosine & 8.060 & 3.157 & 0.39 & $<0.001$ & 0 & 0 & 0 \\
\hline ATP & 424.260 & 162.969 & 0.38 & $<0.001$ & 0 & 0 & 0 \\
\hline
\end{tabular}


Table II. Continued.

\begin{tabular}{lrrcrrrr}
\hline Name of compound & NTs & \multicolumn{1}{c}{ Ts } & Ratio (Ts/NTs) & P-value & ND/all (70) & ND/NTs (35) & ND/Ts (35) \\
\hline Erythrose 4-phosphate & 5.064 & 1.801 & 0.36 & 0.042 & 63 & 34 \\
cis-Aconitic acid & 5.724 & 2.025 & 0.35 & $<0.001$ & 47 & 31 & 16 \\
UTP & 77.543 & 24.624 & 0.32 & $<0.001$ & 3 & 0 & 3 \\
Isocitric acid & 7.347 & 2.204 & 0.30 & $<0.001$ & 46 & 30 \\
2-Oxoisovaleric acid & 5.466 & 1.498 & 0.27 & $<0.001$ & 47 & 30 \\
CTP & 14.088 & 2.946 & 0.21 & $<0.001$ & 28 & 4 & 24
\end{tabular}

ND, not detected; cGMP, cyclic guanosine monophosphate; dATP, deoxyadenosine triphosphate; dCTP, deoxycytidine 5'-triphosphate; dTMP, deoxythymidine monophosphate; dTTP, deoxythymidine triphosphate; Cys, cysteine; GMP, guanosine monophosphate; GABA, gamma-aminobutyric acid; Pro, proline; Asn, asparagine; Tyr, tyrosine; $\beta$-Ala, $\beta$-alanine; Ile, isoleucine; Leu, leucine; Phe, phenylalanine; Asp, aspartic acid; Gly, glycine; Glu, glutamic acid; His, histidine; Val, valine; NADP, nicotinamide adenine dinucleotide phosphate; Thr, threonine; Trp, tryptophan; AMP, adenosine monophosphate; UMP, uridine monophosphate; Lys, lysine; Arg, arginine; GDP, guanosine diphosphate; Ser, serine; IMP, inosine monophosphate; CMP, cytidine monophosphate; PRPP, phosphoribosyl diphosphate; NAD, nicotinamide adenine dinucleotide; dTDP, deoxythymidine diphosphate; Ala, alanine; cAMP, cyclic adenosine monophosphate; ADP, adenosine diphosphate; Gln, glutamine; UDP, uridine diphosphate; GTP, guanosine triphosphate; CDP, cytidine diphosphate; ATP, adenosine triphosphate; UTP, uridine triphosphate; CTP, cytidine triphosphate.

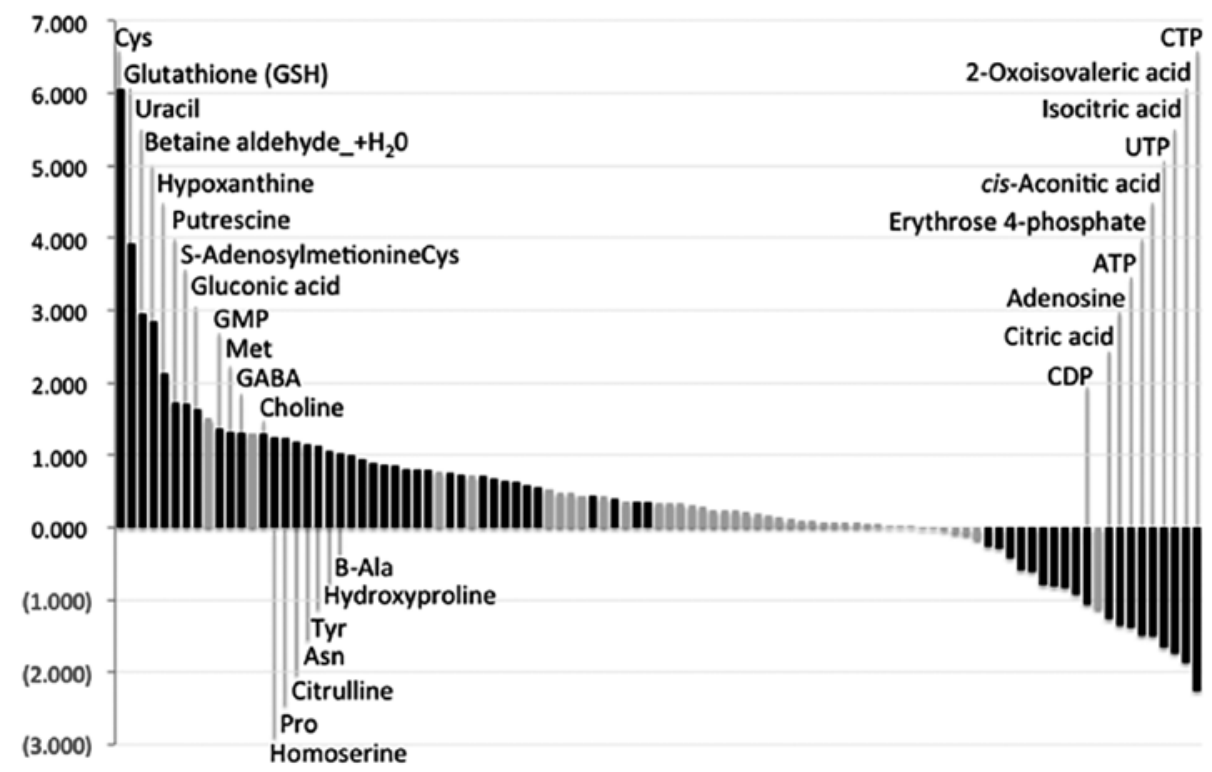

Figure 4. $\log _{2}$ [tumor tissue (Ts)/non-tumor esophageal tissues (NTs)] of metabolites shown in descending order. Metabolites with statistical significance are shown in black. Among these, the names of metabolites are provided when the Ts/NTs ratios were $>2.0$ or $<0.05$.

to the accumulation of overall amino acids in tumor tissues. Glutamine, however, was the only amino acid that was lower in the tumor than the non-tumor tissues. This is presumably due to hyperactive glutamine breakdown, or glutaminolysis, for producing energy and building blocks for continuous proliferation $(33,34)$. In fact, this trend of overall accumulations of amino acids apart from glutamine in tumor regions has been reported elsewhere $(7,8,14)$; accordingly, the near universality of this tumor amino acid profile is intriguing, and the result is reported herein for the first time (at least to the best of our knowledge) for an esophageal tumor.

Few studies have investigated the association between metabolomic characteristics and the pathological status of tumor tissues. However, Wang et al reported 12 key metabolites, such as glucose, AMP, NAD, formate, creatine and choline metabolites that exhibited strong associations with the advancement of esophageal cancer, and are thus likely to be involved in both the carcinogenic process and metastatic alteration of esophageal cancer (14). While attempting to corroborate previous studies, we identified a novel set of metabolites that show significant correlations with the advancement of cancer, such as glycolytic and pentose phosphate pathway intermediates (Table III), taking advantage of CE-TOFMS-based metabolomics, which is best suited to ionic metabolite analysis.

In contrast to glycolytic and pentose phosphate pathway intermediates, the concentrations of citric acid, isocitric acid and malic acid in pT3-4 disease were relatively lower than in pT1-2 disease, suggesting the downregulation of TCA cycle activity in advanced tumors. These results, i.e., a lower TCA 

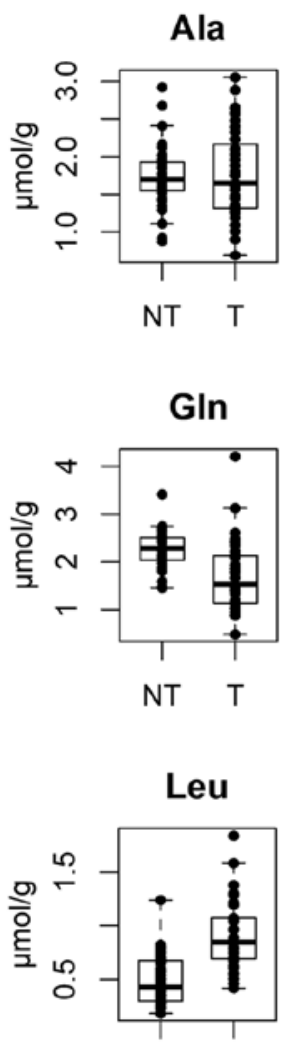

NT T

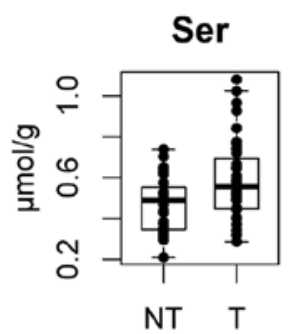

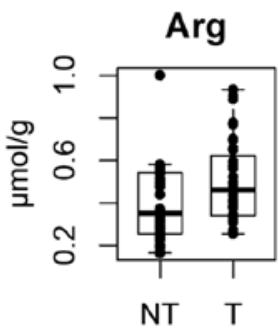

Glu

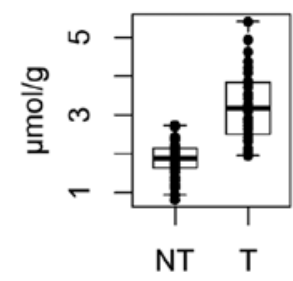

Lys

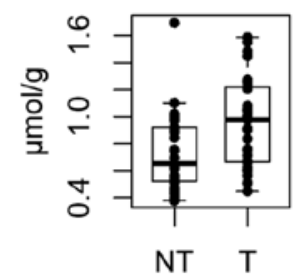

Thr

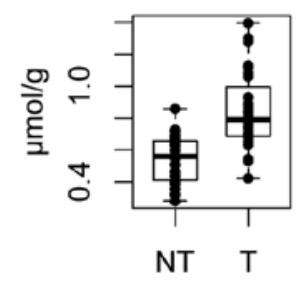

Asn

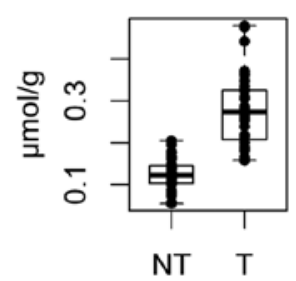

Gly

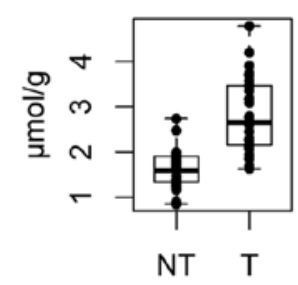

Met

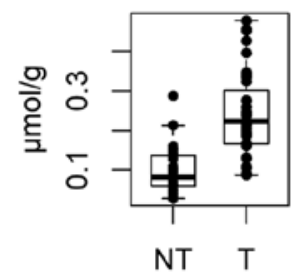

Trp

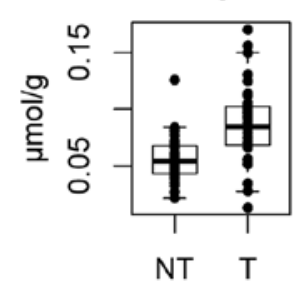

Asp

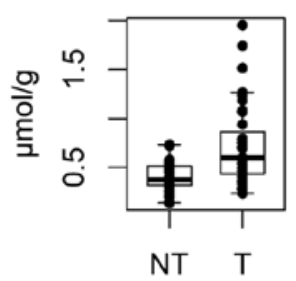

His

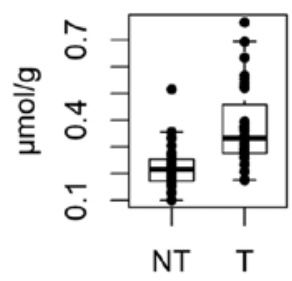

Phe
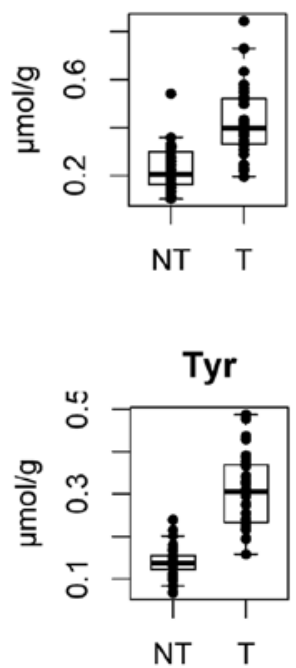

Cys

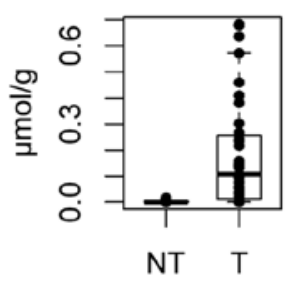

Ile

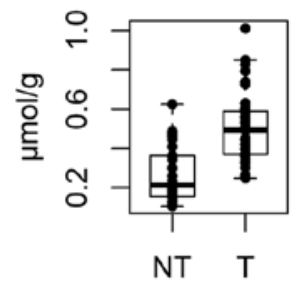

Pro
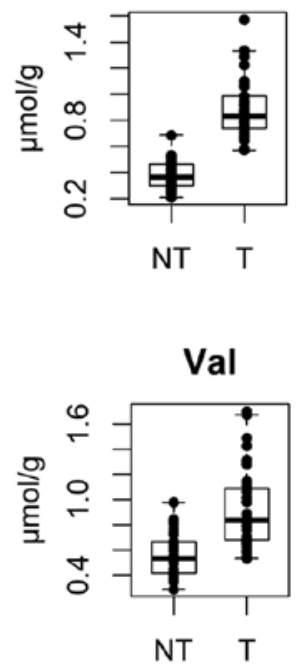

Figure 5. Concentrations of amino acids between tumor tissue (Ts) and non-tumor esophageal tissues (NTs). Ala, alanine; Arg, arginine; Asn, asparagine; Asp, aspartic acid; Cys, cysteine; Gln, glutamine; Glu, glutamic acid; Gly, glycine; His, histidine; Ile, isoleucine; Leu, leucine; Lys, lysine; Met, methionine; Phe, phenylalanine; Pro, proline; Ser, serine; Thr, threonine; Trp, tryptophan; Tyr, tyrosine; Val, valine.

cycle activity and accelerated glycolysis, may be due to a more enhanced Warburg effect in advanced-stage tumors compared with less advanced ones.

A series of nucleotide concentrations were lower in advanced than in less advanced tumors (Table III). Although higher levels of nucleotide metabolites in the advanced tumors were expected, the nucleotide pathway intermediates were mostly lower in the advanced ones. This is possibly due to accelerated utilizations of these nucleotides for their increased DNA synthesis. A lower adenosine monophosphate level in advanced than in less advanced tumors has also been previously reported (14). Total adenylate levels (ATP + ADP + AMP) in pT3-4 (579.8 nmol/g tissue) was almost half of those in pT1-2 $(1096.8 \mathrm{nmol} / \mathrm{g})$, again indicating a higher demand of nucleotides in pT3-4 than in pT1-2 tumor tissues for their increased DNA synthesis. The levels of glutathione and cysteine, two primary anti-oxidants, were on average higher in pT3-4 than in pT1-2, indicating a more reduced status and higher resistance against oxidative stress in pT3-4.
Of note, in cases with $\mathrm{pN}^{+}$, both glutathione and cysteine levels were lower than in cases with $\mathrm{pN}^{-}$, with ratios being 0.50 $(\mathrm{P}=0.130)$ (Table IV) and 0.83 , respectively, translating to a lower resistance against oxidative stress in $\mathrm{pN}^{+}$(note that the ratio of cysteine is not shown in Table IV). Generally, the tumor microenvironment is in a highly oxidative state, and thus, tumor cells tend to be more resistant to oxidative stress. Pavlides et al (35) proposed that stromal tissues rely primarily on glycolysis, producing lactate and ketones, whereas metastatic cancer cells rather use oxidative phosphorylation for energy production, availing the carbon sources provided by the neighboring stromal tissues, and coined the term, 'reverse Warburg effect' $(35,36)$. In this perspective, proliferative tumor regions may contain more cells that mainly use typical Warburg-type energy metabolism, which presumably reduces oxidative stress assuming that oxidative phosphorylation via electron transport chain is a primary source of reactive oxygen species (ROS) (37). By contrast, metastatic tumor cells are rich in mitochondria, producing higher concentrations of ROS, 
Table III. Concentrations of compounds in pT1-2 and pT3-4, and the pT3-4/pT1-2 ratio.

\begin{tabular}{|c|c|c|c|c|}
\hline $\begin{array}{l}\text { Compound } \\
\text { name }\end{array}$ & pT1-2 & pT3-4 & $\begin{array}{c}\text { Ratio } \\
\text { (pT3-4/pT1-2) }\end{array}$ & P-value \\
\hline СТP & 7.968 & 1.459 & 0.18 & 0.022 \\
\hline UTP & 60.532 & 13.984 & 0.23 & 0.651 \\
\hline UDP & 63.988 & 16.823 & 0.26 & 0.088 \\
\hline CDP & 7.582 & 2.133 & 0.28 & 0.030 \\
\hline UMP & 110.416 & 36.403 & 0.33 & 0.010 \\
\hline IMP & 76.817 & 26.164 & 0.34 & 0.046 \\
\hline CMP & 23.980 & 8.466 & 0.35 & 0.007 \\
\hline ATP & 290.826 & 125.085 & 0.43 & 0.406 \\
\hline GTP & 29.731 & 14.475 & 0.49 & 0.143 \\
\hline $\begin{array}{l}\text { 2-Oxoisovaleric } \\
\text { acid }\end{array}$ & 2.399 & 1.231 & 0.51 & 0.033 \\
\hline AMP & 517.276 & 271.559 & 0.52 & 0.019 \\
\hline CoA_divalent & 1.549 & 0.817 & 0.53 & 0.818 \\
\hline $\mathrm{NADP}^{+}$ & 15.262 & 8.773 & 0.57 & 0.112 \\
\hline GDP & 51.221 & 31.083 & 0.61 & 0.009 \\
\hline Citric acid & 182.291 & 112.659 & 0.62 & 0.008 \\
\hline Spermidine & 26.808 & 16.642 & 0.62 & 0.104 \\
\hline Malic acid & 502.515 & 314.098 & 0.63 & 0.015 \\
\hline $\mathrm{NAD}^{+}$ & 228.540 & 143.680 & 0.63 & 0.034 \\
\hline $\mathrm{ADP}$ & 288.720 & 183.195 & 0.63 & 0.104 \\
\hline Sarcosine & 22.653 & 14.786 & 0.65 & 0.017 \\
\hline Isocitric acid & 2.992 & 1.971 & 0.66 & 0.287 \\
\hline $\begin{array}{l}\text { Ribulose } \\
\text { 5-phosphate }\end{array}$ & 26.049 & 40.560 & 1.56 & 0.143 \\
\hline $\begin{array}{l}\text { Ribose } \\
\text { 5-phosphate }\end{array}$ & 5.390 & 8.532 & 1.58 & 0.858 \\
\hline Guanosine & 18.390 & 31.977 & 1.74 & 0.042 \\
\hline $\begin{array}{l}\text { Glutathione } \\
\text { (GSH) }\end{array}$ & 520.187 & 933.978 & 1.80 & 0.356 \\
\hline $\begin{array}{l}\text { Glucose 1- } \\
\text { phosphate }\end{array}$ & 18.228 & 34.979 & 1.92 & 0.923 \\
\hline Tyramine & 0.146 & 0.486 & 3.33 & 0.972 \\
\hline Cys & 64.742 & 217.549 & 3.36 & 0.103 \\
\hline
\end{tabular}

CTP, cytidine triphosphate; UTP, uridine triphosphate; UDP, uridine diphosphate; CDP, cytidine diphosphate; UMP, uridine monophosphate; IMP, inosine monophosphate; CMP, cytidine monophosphate; ATP, adenosine triphosphate; GTP, guanosine triphosphate; AMP, adenosine monophosphate; NADP, nicotinamide adenine dinucleotide phosphate; GDP, guanosine diphosphate; NAD, nicotinamide adenine dinucleotide; ADP, adenosine diphosphate; Cys, cysteine.

and thus may develop a tumor microenvironment with higher oxidative stress $(25,26,36)$. Taken together, the results thus reflect the basal metabolic differences between advanced (but without metastatic) and metastatic tumors.

The present study is limited to the elucidation of the metabolic microenvironment of tissues with or without cancerous cells and may not be suitable for discovery of a potential biomarker for early detection of cancer, as our analysis was
Table IV. Concentrations of compounds in $\mathrm{pN}^{-}$and $\mathrm{pN}^{+}$, and the $\mathrm{pN}^{+} / \mathrm{pN}^{-}$ratio.

\begin{tabular}{|c|c|c|c|c|}
\hline $\begin{array}{l}\text { Compound } \\
\text { name }\end{array}$ & $\mathrm{N}^{-}$ & $\mathrm{N}^{+}$ & $\begin{array}{l}\text { Ratio } \\
\left(\mathrm{N}^{+} / \mathrm{N}^{-}\right)\end{array}$ & P-value \\
\hline CoA_divalent & 2.094 & 0.656 & 0.31 & 0.201 \\
\hline$N, N$-Dimethylglycine & 6.492 & 3.004 & 0.46 & 0.010 \\
\hline ATP & 268.010 & 131.845 & 0.49 & 0.630 \\
\hline Glutathione (GSH) & 1361.444 & 684.717 & 0.50 & 0.130 \\
\hline IMP & 58.624 & 31.555 & 0.54 & 0.027 \\
\hline UTP & 37.779 & 20.726 & 0.55 & 0.280 \\
\hline CDP & 5.071 & 2.877 & 0.57 & 0.374 \\
\hline $\begin{array}{l}\text { Sedoheptulose 7- } \\
\text { phosphate }\end{array}$ & 36.054 & 20.993 & 0.58 & 0.061 \\
\hline UMP & 78.125 & 45.970 & 0.59 & 0.019 \\
\hline Ribulose 5-phosphate & 54.332 & 32.180 & 0.59 & 0.286 \\
\hline Isocitric acid & 3.199 & 1.909 & 0.60 & 0.039 \\
\hline GTP & 25.892 & 15.612 & 0.60 & 0.428 \\
\hline $\begin{array}{l}\text { Fructose } 1,6- \\
\text { diphosphate }\end{array}$ & 94.459 & 57.911 & 0.61 & 0.015 \\
\hline Asp & 1030.898 & 639.629 & 0.62 & 0.041 \\
\hline СТP & 4.155 & 2.588 & 0.62 & 0.830 \\
\hline UDP & 38.597 & 24.347 & 0.63 & 0.056 \\
\hline Ribose 5-phosphate & 10.923 & 6.893 & 0.63 & 0.538 \\
\hline 2-Oxoisovaleric acid & 2.042 & 1.337 & 0.65 & 0.287 \\
\hline Guanine & 30.862 & 46.879 & 1.52 & 0.860 \\
\hline $\begin{array}{l}\text { Phosphoenolpyruvic } \\
\text { acid }\end{array}$ & 2.537 & 4.803 & 1.89 & 0.825 \\
\hline
\end{tabular}

ATP, adenosine triphosphate; IMP, inosine monophosphate; UTP, uridine triphosphate; CDP, cytidine diphosphate; UMP, uridine monophosphate; GTP, guanosine triphosphate; Asp, aspartic acid; GTP, guanosine triphosphate; UDP, uridine diphosphate.

performed using surgically resected specimens and not liquid biopsies. Although not as comprehensive as our study, the metabolomics of biopsy specimens are being realized (38-40). Moreover, once we focus on some specific metabolite markers for pathological tumor status and survival outcome, a minimal amount of tissue, such as a biopsy specimen, may be sufficient for such targeted analysis.

A limitation of this study is that the effects of potential confounding factors affecting the metabolome characteristics, such as the use of chemotherapy and each patient's nutritional status, could not be eliminated. Therefore, the difference in metabolome characteristics between advanced and less-advanced tumors might have been influenced by these confounding factors. Due to the limited number of cases in this study, it would be difficult to exclude the effects of all potential confounding factors completely; however, these effects should be clarified in future trials with sufficient numbers of cases.

In conclusion, in this study, we demonstrated significantly different metabolomic characteristics between tumor and non-tumor tissues of esophageal cancer and identified a novel 
set of metabolites that correlate well with the degree of tumor advancement. This suggests that the pathological disease status and survival outcome may be predicted by analysis of several primary metabolites, possibly even from a biopsy specimen. Further clarification of cancer metabolomics, particularly in relation to the advancement of disease and survival outcome, will enable the selection of more appropriate treatment strategies contributing to individualized medicine.

\section{Acknowledgements}

The authors would like to thank Dr Tamaki Fujimori and Ms. Aya Hoshi, HMT, for their data analysis support. The authors used the English Language Service (International Medical Information Center) for language editing.

\section{Funding}

This study was supported by the Japan Society for the Promotion of Science (JSPS) KAKENHI (Grant no. JP26461998).

\section{Availability of data and materials}

The analyzed datasets generated during the study are available from the corresponding author on reasonable request.

\section{Ethics approval and consent to participate}

The Institutional Review Board of Tokai University (Isehara, Japan) approved the study protocol and all patients provided written informed consent prior to obtaining the samples.

\section{Authors' contributions}

MTo, KK and SO conceived and designed the study; MTo, $\mathrm{KK}$, JO and $\mathrm{AK}$ were involved in data acquisition; KK and YO were involved in data analysis; MTo, KK, SO, HM, MK and MTe were involved in data interpretation. All authors have read and approval the final manuscript.

\section{Consent for publication}

Not applicable.

\section{Competing interests}

The authors declare that they have no competing interests.

\section{References}

1. Domper Arnal MJ, Ferrández Arenas Á and Lanas Arbeloa Á Esophageal cancer: Risk factors, screening and endoscopictreatment in Western and Eastern countries. World J Gastroenterol 21: 7933-7943, 2015.

2. Tachimori Y, Ozawa S, Numasaki H, Fujishiro M, Matsubara H, Oyama T, Shinoda M, Toh Y,Udagawa Hand Uno T: Comprehensive Registry of Esophageal Cancer in Japan, 2008. Esophagus 12: 130-157, 2015.

3. van Hagen P, Hulshof MC, van Lanschot JJ, Steyerberg EW, van Berge Henegouwen MI, Wijnhoven BP, Richel DJ, Nieuwenhuijzen GA, Hospers GA, Bonenkamp JJ, et al; CROSS Group: Preoperative chemoradiotherapy for esophageal or junctional cancer. N Engl J Med 366: 2074-2084, 2012.
4. Ando N, Kato H, Igaki H, Shinoda M, Ozawa S, Shimizu H, Nakamura T, Yabusaki H, Aoyama N, Kurita A, et al: A randomized trial comparing postoperative adjuvant chemotherapy with cisplatin and 5-fluorouracil versus preoperative chemotherapy for localized advanced squamous cell carcinoma of the thoracic esophagus (JCOG9907). Ann Surg Oncol 19: 68-74, 2012

5. Abbassi-Ghadi N, Kumar S, Huang J, Goldin R, Takats Z and Hanna GB: Metabolomic profiling of oesophago-gastric cancer: A systematic review. Eur J Cancer 49: 3625-3637, 2013.

6. Pavlova NN and Thompson CB: The emerging hallmarks of cancer metabolism. Cell Metab 23: 27-47, 2016.

7. Kami K, Fujimori T, Sato H, Sato M, Yamamoto H, Ohashi Y, Sugiyama N,Ishihama Y,Onozuka H,Ochiai A, et al: Metabolomic profiling of lung and prostate tumor tissues by capillary electrophoresis time-of-flight mass spectrometry. Metabolomics 9: 444-453, 2013.

8. Hirayama A, Kami K, Sugimoto M, Sugawara M, Toki N Onozuka H, Kinoshita T, Saito N, Ochiai A, Tomita M, et al: Quantitative metabolome profiling of colon and stomach cancer microenvironment by capillary electrophoresis time-of-flight mass spectrometry. Cancer Res 69: 4918-4925, 2009.

9. Ikeda A, Nishiumi S, Shinohara M, Yoshie T, Hatano N, Okuno T, Bamba T, Fukusaki E, Takenawa T, Azuma T, et al: Serum metabolomics as a novel diagnostic approach for gastrointestinal cancer. Biomed Chromatogr 26: 548-558, 2012.

10. Xu J, Chen Y, Zhang R, Song Y, Cao J, Bi N, Wang J, He J, Bai J, Dong L, et al: Global and targeted metabolomics of esophageal squamous cell carcinoma discovers potential diagnostic and therapeutic biomarkers. Mol Cell Proteomics 12: 1306-1318, 2013.

11. Zhang X, Xu L, Shen J, Cao B, Cheng T, Zhao T, Liu X and Zhang H: Metabolic signatures of esophageal cancer: NMR-based metabolomics and UHPLC-based focused metabolomics of blood serum. Biochim Biophys Acta 1832: 1207-1216, 2013.

12. Ma H,Hasim A, Mamtimin B, Kong B,Zhang HP and Sheyhidin I: Plasma free amino acid profiling of esophageal cancer using high-performance liquid chromatography spectroscopy. World J Gastroenterol 20: 8653-8659, 2014.

13. Yang Y, Wang L, Wang S, Liang S, Chen A, Tang H, Chen L and Deng F: Study of metabonomic profiles of human esophageal carcinoma by use of high-resolution magic-angle spinning $1 \mathrm{H}$ NMR spectroscopy and multivariate data analysis. Anal Bioanal Chem 405: 3381-3389, 2013.

14. Wang L, Chen J, Chen L, Deng P, Bu Q, Xiang P, Li M, Lu W, $\mathrm{Xu} \mathrm{Y}$, Lin $\mathrm{H}$, et al: $1 \mathrm{H}-\mathrm{NMR}$ based metabonomic profiling of human esophageal cancer tissue. Mol Cancer 12: 25, 2013.

15. Wu H, Xue R, Lu C, Deng C, Liu T, Zeng H, Wang Q and Shen X: Metabolomic study for diagnostic model of oesophageal cancer using gas chromatography/mass spectrometry. J Chromatogr B Analyt Technol Biomed Life Sci 877: 3111-3117, 2009.

16. The Japan Eshophageal Society: Japanese Classification of Esophageal Cancer 11th edition. Esophagus: Nov 10, 2016 (Epub ahead of print).

17. Ohashi Y, Hirayama A, Ishikawa T, Nakamura S, Shimizu K, Ueno Y, Tomita M and Soga T: Depiction of metabolome changes in histidine-starved Escherichia coli by CE-TOFMS. Mol Biosyst 4: 135-147, 2008.

18. Ooga T, Sato H, Nagashima A, Sasaki K, Tomita M, Soga T and Ohashi Y: Metabolomic anatomy of an animal model revealing homeostatic imbalances in dyslipidaemia. Mol Biosyst 7: 1217-1223, 2011.

19. Sugimoto M, Wong DT, Hirayama A, Soga T and Tomita M: Capillary electrophoresis mass spectrometry-based saliva metabolomics identified oral, breast and pancreatic cancer-specific profiles. Metabolomics 6: 78-95, 2010.

20. Junker BH, Klukas C and Schreiber F: VANTED: A system for advanced data analysis and visualization in the context of biological networks. BMC Bioinformatics 7: 109, 2006.

21. Kawamura T, Kusakabe T, Sugino T, Watanabe K, Fukuda T, Nashimoto A, Honma K and Suzuki T: Expression of glucose transporter-1 in human gastric carcinoma: Association with tumor aggressiveness, metastasis, and patient survival. Cancer 92: 634-641, 2001.

22. Hamabe A, Yamamoto H, Konno M, Uemura M, Nishimura J, Hata T, Takemasa I, Mizushima T, Nishida N, Kawamoto K, et al: Combined evaluation of hexokinase 2 and phosphorylated pyruvate dehydrogenase-E1 $\alpha$ in invasive front lesions of colorectal tumors predicts cancer metabolism and patient prognosis. Cancer Sci 105: 1100-1108, 2014. 
23. Fukuda S, Miyata H, Miyazaki Y, Makino T, Takahashi T, Kurokawa Y, Yamasaki M, Nakajima K, Takiguchi S, Mori M, et al: Pyruvate kinase M2 modulates esophageal squamous cell carcinoma chemotherapy response by regulating the pentose phosphate pathway. Ann Surg Oncol 22 (Suppl 3): S1461-S1468, 2015.

24. Hockel M, Schlenger K, Aral B, Mitze M, Schaffer U and Vaupel P: Association between tumor hypoxia and malignant progression in advanced cancer of the uterine cervix. Cancer Res 56: 4509-4515, 1996.

25. Payen VL, Porporato PE, Baselet B and Sonveaux P: Metabolic changes associated with tumor metastasis, part 1: Tumor $\mathrm{pH}$, glycolysis and the pentose phosphate pathway. Cell Mol Life Sci 73: 1333-1348, 2016.

26. Porporato PE, Payen VL, Baselet B and Sonveaux P: Metabolic changes associated with tumor metastasis, part 2: Mitochondria, lipid and amino acid metabolism. Cell Mol Life Sci 73: 1349-1363, 2016.

27. Jin H, Qiao F, Chen L, Lu C, Xu L and Gao X: Serum metabolomic signatures of lymph node metastasis of esophageal squamous cell carcinoma. J Proteome Res 13: 4091-4103, 2014.

28. Warburg O: On the origin of cancer cells. Science 123: 309-314, 1956.

29. Honjo H, Kaira K, Miyazaki T, Yokobori T, Kanai Y, Nagamori S, Oyama T, Asao T and Kuwano H: Clinicopathological significance of LAT1 and ASCT2 in patients with surgically resected esophageal squamous cell carcinoma. J Surg Oncol 113: 381-389, 2016.

30. Kobayashi H, Ishii Y and Takayama T: Expression of L-type amino acid transporter 1 (LAT1) in esophageal carcinoma. J Surg Oncol 90: 233-238, 2005.

31. Younes M,Pathak M, Finnie D, Sifers RN,Liu Y and Schwartz MR: Expression of the neutral amino acids transporter ASCT1 in esophageal carcinomas. Anticancer Res 20: 3775-3779, 2000.
32. Morselli E, Galluzzi L, Kepp O, Vicencio JM, Criollo A, Maiuri MC and Kroemer G: Anti- and pro-tumor functions of autophagy. Biochim Biophys Acta 1793: 1524-1532, 2009.

33. Vander Heiden MG, Cantley LC and Thompson CB: Understanding the Warburg effect: The metabolic requirements of cell proliferation. Science 324: 1029-1033, 2009.

34. Tsun ZY and Possemato R: Amino acid management in cancer. Semin Cell Dev Biol 43: 22-32, 2015.

35. Pavlides S, Whitaker-Menezes D, Castello-Cros R, Flomenberg N, Witkiewicz AK, Frank PG, Casimiro MC, Wang C, Fortina P, Addya S, et al: The reverse Warburg effect: Aerobic glycolysis in cancer associated fibroblasts and the tumor stroma. Cell Cycle 8: 3984-4001, 2009.

36. Sotgia F, Whitaker-Menezes D, Martinez-Outschoorn UE, Flomenberg N, Birbe RC, Witkiewicz AK, Howell A, Philp NJ, Pestell RG and Lisanti MP: Mitochondrial metabolism in cancer metastasis: Visualizing tumor cell mitochondria and the 'reverse Warburg effect' in positive lymph node tissue. Cell Cycle 11: 1445-1454, 2012

37. Orrenius S: Reactive oxygen species in mitochondria-mediated cell death. Drug Metab Rev 39: 443-455, 2007.

38. Benahmed MA, Elbayed K, Daubeuf F, Santelmo N, Frossard N and Namer IJ: NMR HRMAS spectroscopy of lung biopsy samples: Comparison study between human, pig, rat, and mouse metabolomics. Magn Reson Med 71: 35-43, 2014.

39. Li M, Song Y, Cho N, Chang JM, Koo HR, Yi A, Kim H, Park S and Moon WK: An HR-MAS MR metabolomics study on breast tissues obtained with core needle biopsy. PLoS One 6: e25563, 2011.

40. Choi JS, Baek HM, Kim S, Kim MJ, Youk JH, Moon HJ, Kim EK and Nam YK: Magnetic resonance metabolic profiling of breast cancer tissue obtained with core needle biopsy for predicting pathologic response to neoadjuvant chemotherapy. PLoS One 8: e83866, 2013. 